\title{
Circuit Covers of Signed Eulerian Graphs
}

\author{
Bo Bao Rong Chen* Genghua Fan ${ }^{\dagger}$ \\ Center for Discrete Mathematics \\ Fuzhou University \\ Fuzhou, P. R. China \\ tomcat0830@163.com, \{rongchen,fan\}@fzu.edu.cn
}

Submitted: Oct 22, 2019; Accepted: Dec 7, 2020; Published: Jan 29, 2021

(c) The authors. Released under the CC BY-ND license (International 4.0).

\begin{abstract}
A signed circuit cover of a signed graph is a natural analog of a circuit cover of a graph, and is equivalent to a covering of its corresponding signed-graphic matroid with circuits. It was conjectured that a signed graph whose signed-graphic matroid has no coloops has a 6-cover. In this paper, we prove that the conjecture holds for signed Eulerian graphs.
\end{abstract}

Mathematics Subject Classifications: 05C21, 05C22

\section{Introduction}

Let $G$ be a graph. A signed graph is a pair $(G, \Sigma)$ with $\Sigma \subseteq E(G)$, each edge in $\Sigma$ is labelled by -1 and other edges are labelled by 1 . The graph $G$ can be viewed as the signed graph $(G, \varnothing)$. A circuit is a connected 2-regular graph. A circuit $C$ of $G$ is balanced if $|C \cap \Sigma|$ is even, otherwise it is unbalanced. We say that a subgraph of $(G, \Sigma)$ is unbalanced if it contains an unbalanced circuit, otherwise it is balanced. Signed graphs is a special class of "biased graphs", which was defined by Zaslavsky in [7,8]. Just as biased graphs, there are two interesting classes of matroids, the class of signed-graphic matroids and the class of even-cycle matroids, associated with signed graphs, which in fact are special classes of "frame matroids" and "lifted-graphic matroids" associated with biased graphs, respectively.

A barbell is a union of two unbalanced circuits sharing exactly one vertex or a union of two vertex-disjoint unbalanced circuits together with a minimal path joining them. A signed circuit of $(G, \Sigma)$ is a balanced circuit or a barbell. We say the matroid with $E(G)$

*Supported by NSFC (No. 11971111) and NSFFP (No. 2019J01645).

${ }^{\dagger}$ Supported by NSFC (No. 11971110). 
as its ground set and with the set of all signed circuits as its circuit set is the signedgraphic matroid defined on $(G, \Sigma)$. We say that $(G, \Sigma)$ is flow-admissible if each element of $E(G)$ is in a circuit of its signed-graphic matroid, that is, each edge of $G$ is in a signed circuit of $(G, \Sigma)$.

For a positive integer $k$, we say that a signed graph $(G, \Sigma)$ has a $k$-cover if there is a family $\mathcal{C}$ of signed circuits of $(G, \Sigma)$ such that each edge of $G$ belongs to exactly $k$ members of $\mathcal{C}$. For ordinary graphs $G$ (signed graph $(G, \Sigma)$ with $\Sigma=\varnothing$ ), a $k$-cover of $G$ is just a family of circuits which together covers each edge of $G$ exactly $k$ times. In [1], Bermond, Jackson and Jaeger proved that every bridgeless graph $G$ has a 4-cover. Fan [4] proved that every bridgeless graph $G$ has a 6 -cover. Together it follows that every bridgeless graph $G$ has a k-cover, for every even integer $k$ greater than 2 . The only left case that $k=2$ is the famous Circuit Double Cover Conjecture: every bridgeless graph $G$ has a 2-cover, which is still open and believed to be very hard. It is somehow a surprise that it is even unknown whether there is an integer $k$ such that every signed graph $(G, \Sigma)$ has a $k$-cover.

Let $A$ and $B$ be two vertex-disjoint unbalanced circuits of length $2 m+1$. Let $G$ be the signed graph obtained from $A$ and $B$ by joining $A$ and $B$ with two internally disjoint paths of length $2 m+1$ such that the two paths form an unbalanced circuit. Then each signed circuit of $G$ is a barbell of $6 m+3$ edges. Any $k$-cover of $G$ contains $k|E(G)|=k(8 m+4)=4 k(2 m+1)$ edges, which must be divisible by $6 m+3=3(2 m+1)$. That is, $4 k$ must be divisible by 3 , which means that $k$ cannot be 2 or 4 . Thus $G$ has neither 2-covers nor 4-covers. Consider the singed graph $H$ consisting of three unbalanced circuits of length $2 m+1$ with exactly one vertex in common. Then each signed circuit of $H$ is a barbell of $4 m+2$ edges. Any $k$-cover of $H$ contains $k|E(G)|=k(6 m+3)=3 k(2 m+1)$ edges, which must be divisible by $4 m+2=2(2 m+1)$. That is, $3 k$ must be divisible by 2 , which means that $k$ cannot be odd. Thus $H$ has no $k$-cover for any odd $k$. These counterexamples were first given by Fan [5], who also proposed the following conjecture.

Conjecture 1.1. Every flow-admissible signed graph has a 6-cover.

In this paper, we prove

Theorem 1.2. Conjecture 1.1 holds for signed Eulerian graphs.

In [3], Cheng, Lu, Luo, and Zhang proved that each signed Eulerian graph with an even number of negative edges has a 2-cover. We will prove Theorem 1.2 from a different aspect, and our proof does not rely on their result.

This paper is organised as follows. Definitions and results needed in the proof of Theorem 1.2 are given in Section 2. Theorem 1.2 will be proved in Section 4 by contradiction. All "small" signed Eulerian graphs occurring in Section 4 in the proof by contradiction are dealt with in Section 3.

\section{Preliminaries}

Let $G$ be a finite graph. Let loops $(G)$ denote the set of loops in $G$. Let $\Delta(G)$ and $\delta(G)$ be the maximal and minimal degree of $G$, respectively. For a positive integer $k$, let $V_{k}(G)$ be 
the subset of $V(G)$ consisting of degree- $k$ vertices of $G$. A subgraph $H$ of $G$ is spanning if $V(H)=V(G)$. In this paper, we will also use $H$ to denote its edge-set. For example, we will let $G \backslash H$ denote $G \backslash E(H)$. If exactly one component of $G$ has edges, then we say that $G$ is connected up to isolated vertices. Evidently, a connected graph is also connected up to isolated vertices, but the converse maybe not true.

We say that $G$ is even if every vertex of $G$ is of even degree. If an even graph is connected, we say that it is Eulerian. A circuit $C$ of $G$ is non-separating if $G \backslash C$ is connected, otherwise, it is separating. A theta graph is a graph that consists of a pair of vertices joined by three internally vertex-disjoint paths. Let $\mathcal{C}$ be a circuit-decomposition of an Eulerian graph $G$. Let $H$ be a graph with $\mathcal{C}$ as its vertex set, where two vertices in $H$ are adjacent if and only if their corresponding circuits in $G$ have a common vertex. We say that $H$ is determined by $\mathcal{C}$.

Lemma 2.1. Let $G$ be an Eulerian graph with $\Delta(G) \geqslant 4$. Let $C$ be a circuit of $G$. Then there is a circuit $C^{\prime}$ of $G$ with $C \cap C^{\prime}=\varnothing$ such that $G \backslash C^{\prime}$ is connected up to isolated vertices.

Proof. Since $G$ is Eulerian, $G$ has a circuit-decomposition $\mathcal{C}$ containing $C$. Let $H$ be the graph determined by $\mathcal{C}$. Since $G$ is connected with $\Delta(G) \geqslant 4$, the graph $H$ is connected with at least two vertices. Let $T$ be a spanning tree of $H$. Since $T$ has at least two degree-1 vertex, $T$ has a degree-1 vertex, say $C^{\prime}$, which is not $C$. Then $C^{\prime}$ is the circuit as required by the lemma.

Lemma 2.2. Let $G$ be a 2-connected graph with $|V(G)| \geqslant 3$. For any vertex $v$ of $G$, there is an edge e of $G-v$ such that $G-V(e)$ is connected.

Proof. Let $C$ be a circuit of $G$ passing through $v$ with $|C|$ as large as possible. Evidently, $|C| \geqslant 3$ as $|V(G)| \geqslant 3$ and $G$ is 2-connected. Let $e$ be an edge of $C$ that is not incident with $v$. Then $G-V(e)$ is connected, otherwise we can find a longer circuit going through $v$.

A set $\Sigma^{\prime} \subseteq E(G)$ is a signature of $(G, \Sigma)$ if $(G, \Sigma)$ and $\left(G, \Sigma^{\prime}\right)$ have the same balanced circuits and the same unbalanced circuits. Evidently, for any edge-cut $C^{*}$ of $G$, the symmetric difference $\Sigma \triangle C^{*}$ is a signature of $(G, \Sigma)$. We say that $\left(G, \Sigma^{\prime}\right)$ is obtained from $(G, \Sigma)$ by switching. The following three lemmas are well-known results on signed graphs, which will be frequently used in Section 3 without reference. Please refer to ( [2], Lemma 3.5.), if the reader needs more detail about Lemma 2.3.

Lemma 2.3. All edges of a balanced signed subgraph of $(G, \Sigma)$ can be labelled by 1 by switching.

Lemma 2.4. Each signed theta-graph has a balanced circuit and can not have exactly two balanced circuits.

Lemma 2.5. Every 2-edge-connected signed graph containing two edge-disjoint unbalanced circuits is flow-admissible. 
In ( [6], Theorem 4.2.), Máčajová and Škoviera proved that a flow-admissible signed Eulerian graph with an odd number of negative edges contains three edge-disjoint unbalanced circuits. On the other hand, since each unbalanced Eulerian signed graph with an even number of negative edges contains two edge-disjoint unbalanced circuits, we have

Lemma 2.6. A flow-admissible unbalanced signed Eulerian graph contains two edgedisjoint unbalanced circuits.

For simplicity, we will also use $G$ to denote a signed graph defined on $G$.

\section{Signed Eulerian graphs with special circuit decompositions}

Let $k$ be a positive integer. Let $k G$ be the graph obtained from $G$ by replacing each edge in $G$ with exactly $k$ parallel edges. Consider a graph constructed as follows. For $k \geqslant 3$, let $G$ be a circuit of length $k$ and $N$ be a subdivision of $2 G$. Let $C$ be a circuit of $N$, we say that $C$ is small if $\left|V(C) \cap V_{4}(N)\right|=2$, otherwise, $C$ is long. When $C$ is small, we also say that each vertex in $V(C) \cap V_{4}(N)$ is an end of $C$. Let $e_{1}, e_{2}$ be edges in a small circuit of $N$ such that $\left\{e_{1}, e_{2}\right\}$ is not an edge-cut of $N$. That is, $\left\{e_{1}, e_{2}\right\}$ separates the two ends of the small circuit. We say that the signed graph obtained from $N$ by labelling $\left\{e_{1}, e_{2}\right\}$ by -1 and all other edges by 1 is a necklace of length $k$. Evidently, all small circuits in a necklace are balanced and all long circuits are unbalanced. Hence, the small circuits form a 1-cover in a necklace.

In the rest of this section, we will always let $G$ denote a 2-connected flowadmissible signed Eulerian graph with $\delta(G) \geqslant 4$, and $\mathcal{C}$ a circuit-decomposition of $\boldsymbol{G}$, and let $\boldsymbol{H}$ be the graph determined by $\mathcal{C}$. We say that $\mathcal{C}$ is optimal if it satisfies the following properties:

$(\mathrm{CD} 1) \mathcal{C}$ is chosen with the number of unbalanced circuits as large as possible.

$(\mathrm{CD} 2)$ subject to $(\mathrm{CD} 1), \mathcal{C}$ is chosen with $|\mathcal{C}|$ as large as possible.

In the rest of this section, we will always assume that $\mathcal{C}$ is optimal. For any $C \in \mathcal{C}$, we say that $C$ is a balanced vertex of $H$ if $C$ is a balanced circuit of $G$, otherwise it is unbalanced.

The following lemma follows immediately from (CD1), (CD2), and Lemma 2.6.

Lemma 3.1. For every pair of adjacent vertices $C_{i}$ and $C_{j}$ in $H$, if $C_{i}$ is balanced, we have

1. $1 \leqslant\left|V_{G}\left(C_{i}\right) \cap V_{G}\left(C_{j}\right)\right| \leqslant 2$,

2. $C_{i} \cup C_{j}$ is balanced when $C_{j}$ is balanced, and

3. $C_{i} \cup C_{j}$ is not flow-admissible when $C_{j}$ is unbalanced.

Lemma 3.2. For every pair of adjacent unbalanced vertices $C_{i}$ and $C_{j}$ in $H$, if $\mid V_{G}\left(C_{i}\right) \cap$ $V_{G}\left(C_{j}\right) \mid \geqslant 3$, then $C_{i} \cup C_{j}$ is a necklace. 
Proof. Since $C_{i}$ and $C_{j}$ are unbalanced, for any circuit decomposition $\mathcal{C}^{\prime}$ of $C_{i} \cup C_{j}$, either all circuits in $\mathcal{C}^{\prime}$ are balanced or at least two of them are unbalanced. If $C_{i} \cup C_{j}$ has an unbalanced circuit avoiding some vertex in $V_{4}\left(C_{i} \cup C_{j}\right)$, then $C_{i} \cup C_{j}$ can be decomposed into at least three circuits and two of which are unbalanced, which is not possible as $\mathcal{C}$ is optimal. So each circuit in $C_{i} \cup C_{j}$ avoiding a vertex in $V_{4}\left(C_{i} \cup C_{j}\right)$ is balanced. Hence, $C_{i} \cup C_{j}$ is a necklace.

We say that $G$ is cover-decomposable if $G$ can be decomposed into two proper edgedisjoint flow-admissible signed Eulerian subgraphs.

Lemma 3.3. If $H$ is isomorphic to a graph pictured as Figure 1 and $G$ has no balanced loops, then $G$ is cover-decomposable or has a 6 -cover.

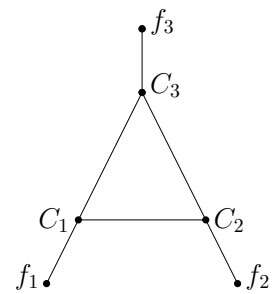

(a)

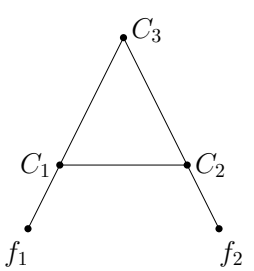

(b)

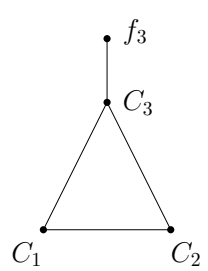

(c)

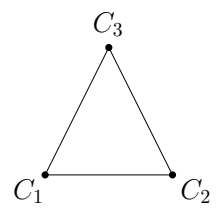

$(d)$

Figure 1: All degree-3 vertices are balanced, and others are unbalanced. All $f_{i}$ 's are loops of $G$.

Proof. Assume otherwise. Assume that $H$ is isomorphic to the graph pictured as Figure 1 (d). For any $1 \leqslant i<j \leqslant 3$, when $\left|V\left(C_{i}\right) \cap V\left(C_{j}\right)\right| \leqslant 2$, it is obvious that $C_{i} \cup C_{j}$ has a 1-cover; when $\left|V\left(C_{i}\right) \cap V\left(C_{j}\right)\right| \geqslant 3$, it follows from Lemma 3.2 that $C_{i} \cup C_{j}$ is a necklace, so $C_{i} \cup C_{j}$ has a 1-cover too. Then $G$ has a 2-cover. So $H$ is isomorphic to a graph pictured as Figure 1 (a)-(c). Note that, $1 \leqslant\left|V_{G}\left(C_{i}\right) \cap V_{G}\left(C_{j}\right)\right| \leqslant 2$ when $C_{i}$ is balanced by Lemma 3.1. When some $C_{i}$ is a loop, implying that $G$ is isomorphic to the graph pictured as Figure 1 (b) or (c), since each pair of adjacent circuits intersect in at most 2 vertices, there are a few cases to check that $G$ has a 6 -cover. So no $C_{i}$ is a loop. When $\left|V_{G}\left(C_{i}\right) \cap V_{G}\left(C_{j}\right)\right|=1$ for all $1 \leqslant i<j \leqslant 3$, since $C_{1} \cup C_{2} \cup C_{3}$ is isomorphic to a $2 K_{3}$-subdivision, combined the fact that all $f_{i}$ are unbalanced loops, it is easy to see that $G$ has a 6 -cover. Hence, $\left|V_{G}\left(C_{i}\right) \cap V_{G}\left(C_{j}\right)\right| \geqslant 2$ for some $1 \leqslant i<j \leqslant 3$.

Assume that $H$ is isomorphic to a graph pictured as Figure 1 (a). By Lemma 3.1 (1) and symmetry we may assume that $V_{G}\left(C_{2}\right) \cap V_{G}\left(C_{3}\right)=\{u, v\}$. Let $C$ be the circuit of $C_{2} \cup C_{3}$ that is incident to neither $f_{2}$ nor $f_{3}$. Since $C$ is balanced by Lemma 3.1 (2), $G \backslash C$ is not connected otherwise $G$ is cover-decomposable, so $V_{G}\left(C_{1}\right) \cap V_{G}\left(C_{2} \cup C_{3}\right) \subseteq$ $V_{G}(C)-\{u, v\}$. When $\left|V_{G}\left(C_{1}\right) \cap V_{G}\left(C_{2}\right)\right|=\left|V_{G}\left(C_{1}\right) \cap V_{G}\left(C_{3}\right)\right|=1$, the graph $G$ has a 2-cover. When $\left|V_{G}\left(C_{1}\right) \cap V_{G}\left(C_{i}\right)\right|=2$ for some $2 \leqslant i \leqslant 3$, since $G \backslash$ loops $(G)$ is balanced by Lemma 3.1 (2), there is a non-separating balanced circuit $C^{\prime}$ contained in $C \cup C_{i}$, 
implying that $G$ is cover-decomposable. Hence, $H$ is isomorphic to a graph pictured as Figure 1 (b) or (c).

Assume that $V_{G}\left(C_{2}\right) \cap V_{G}\left(C_{3}\right)=\{u, v\}$. Since exactly one of $\left\{C_{2}, C_{3}\right\}$ is unbalanced, there is a $(u, v)$-path $P$ of $C_{2} \cup C_{3}$ such that a circuit in $C_{2} \cup C_{3}$ is unbalanced if and only if it contains $P$. Since all degree-3 vertices in Figure 1 are balanced, $P$ is not incident to $f_{2}$ or $f_{3}$. Let $C$ the unique balanced circuit of $C_{2} \cup C_{3}$ that is not incident to $f_{2}$ or $f_{3}$. Since $\left(C_{2} \cup C_{3}\right)-C$ and $f_{3}$ are unbalanced, $G \backslash C$ is not connected, so $V_{G}\left(C_{1}\right) \cap V_{G}\left(C_{2} \cup C_{3}\right) \subseteq V_{G}(C)-\{u, v\}$. When $C_{1} \cup C_{2}$ is a necklace, implying that $H$ is isomorphic to a graph pictured as Figure 1 (c) by Lemma 3.1, there is a non-separating small circuit $C^{\prime}$ of the necklace $C_{1} \cup C_{2}$ with $C^{\prime} \subseteq C_{1} \cup\left(C_{2}-P\right)$. Since $\left(C_{2} \cup C_{3}\right)-C$ and $f_{3}$ are unbalanced, $G \backslash C^{\prime}$ is flow-admissible, so $G$ is cover-decomposable as $C^{\prime}$ is balanced. Hence, by Lemma 3.1 (1) or Lemma 3.2, we have $\left|V_{G}\left(C_{1}\right) \cap V_{G}\left(C_{i}\right)\right| \leqslant 2$ for each $2 \leqslant i \leqslant 3$. Moreover, since $V_{G}\left(C_{1}\right) \cap V_{G}\left(C_{2} \cup C_{3}\right) \subseteq V_{G}(C)-\{u, v\}$, repeatedly using a similar strategy, we can find a 6 -cover of $G$ or a non-separating balanced circuit $C$ such that $G \backslash C$ is flow-admissible, a contradiction.

By symmetry we may therefore assume that $\left|V_{G}\left(C_{i}\right) \cap V_{G}\left(C_{3}\right)\right|=1$ for each $1 \leqslant i \leqslant 2$. Set $m=\left|V_{G}\left(C_{1}\right) \cap V_{G}\left(C_{2}\right)\right| \geqslant 2$. When $m=2$, by simple computation, the lemma holds. So $m \geqslant 3$. By Lemmas 3.1 and 3.2, $H$ is isomorphic to the graph pictured as Figure 1 (c) and $C_{1} \cup C_{2}$ is a necklace of length $m$. Assume that $G$ is a counterexample to the lemma with $|V(G)|$ as small as possible. When $C_{3}$ does not share a vertex with a small circuit $C$ of $C_{1} \cup C_{2}$, delete $C$ and identify its two ends as a new vertex. Let $G^{\prime}$ be the new graph. Then $G^{\prime}$ is cover-decomposable or has a 6 -cover by the choice of $G$, so is $G$ since $C$ is balanced. Hence, $C_{3}$ intersects all small circuits of $C_{1} \cup C_{2}$. Moreover, since $m \geqslant 3$ and $\left|V_{G}\left(C_{i}\right) \cap V_{G}\left(C_{3}\right)\right|=1$ for each $1 \leqslant i \leqslant 2$, there are edge-disjoint long circuits $C_{1}^{\prime}, C_{2}^{\prime}$ of $C_{1} \cup C_{2}$ with $\left|V_{G}\left(C_{1}^{\prime}\right) \cap V_{G}\left(C_{3}\right)\right|=2$ and $\left|V_{G}\left(C_{2}^{\prime}\right) \cap V_{G}\left(C_{3}\right)\right| \geqslant 1$. Since $C_{1}^{\prime}, C_{2}^{\prime}$ are unbalanced and $C_{1}^{\prime} \cup C_{2}^{\prime}=C_{1} \cup C_{2}$, the graph determined by $\left\{C_{1}^{\prime}, C_{2}^{\prime}, C_{3},\left\{f_{3}\right\}\right\}$ isomorphic to the graph pictured as Figure 1 (c). Since $\left|V_{G}\left(C_{1}^{\prime}\right) \cap V_{G}\left(C_{3}\right)\right|=2$, the lemma holds by similar analysis in the second paragraph of the proof.

Let $C$ be a separating circuit of a graph $G$ with $u, v \in V(C)$. Let $P$ be an $(u, v)$-path on $C$. For a component $G^{\prime}$ of $G \backslash C$, if $V\left(G^{\prime}\right) \cap V(P) \neq \varnothing$ we say that $G^{\prime}$ intersects $P$; if $V\left(G^{\prime}\right) \cap V(C) \subseteq V(P)-\{u, v\}$ we say that $G^{\prime}$ properly intersects with $P$.

Lemma 3.4. Let $C$ be a separating circuit of $G$ such that all components of $G \backslash C$ are unbalanced. Let $C^{\prime}$ be a circuit-component of $G \backslash C$ with $\{u, v\}=V(C) \cap V\left(C^{\prime}\right)$. Let $P_{1}$ and $P_{2}$ be the $(u, v)$-paths of $C$. When $C$ is balanced or $G \backslash C$ has at least three components, one of the following holds.

(1) $G$ is cover-decomposable, or

(2) $G \backslash C$ has exactly three components, none of which is flow-admissible and one of which properly intersects with $P_{i}$ for each $1 \leqslant i \leqslant 2$.

Proof. Assume that (1) is not true. Without loss of generality we may assume that $C^{\prime}=\{e, f\}$. Since $C^{\prime}$ is unbalanced, we may assume that $P_{1} \cup\{e\}$ and $P_{2} \cup\{x\}$ are 
balanced for some $x \in\{e, f\}$. Since $G \backslash C$ has at least two components, besides $C^{\prime}$, some component of $G \backslash C$ intersects with some $P_{i}$, say $P_{2}$. Since $C$ is balanced or $G \backslash C$ has at least three components, $G \backslash\left(P_{1} \cup\{e\}\right)$ has two edge-disjoint unbalanced circuits. Since (1) does not hold, $G \backslash\left(P_{1} \cup\{e\}\right)$ is disconnected, so there exists some non-flow-admissible component of $G \backslash C$ properly intersecting with $P_{1}$. Repeating the analysis, there is also a non-flow-admissible component of $G \backslash C$ properly intersecting with $P_{2}$. So $G \backslash C$ has at least three components.

Let $G_{i}$ be the union of the components of $G \backslash C$ that properly intersects with $P_{i}$ for each $1 \leqslant i \leqslant 2$. Then $G_{1}$ and $G_{2}$ are not flow-admissible. Assume that $G_{1}$ is disconnected. Since $G_{1}$ contains two edge-disjoint unbalanced circuits, $G_{1} \cup P_{1} \cup\{x\}$ and $G \backslash\left(G_{1} \cup P_{1} \cup\right.$ $\{x\}$ ) are flow-admissible, implying that (1) holds. Hence, $G_{1}$ is connected, so is $G_{2}$ by symmetry. Besides $C^{\prime}, G_{1}$ and $G_{2}$, assume that $G \backslash C$ has another component $G_{3}$. Since $G_{3}$ is unbalanced and intersects $V\left(P_{1}\right)$ and $V\left(P_{2}\right)$ by the definition of $G_{1}$ and $G_{2}$ and the fact that $G$ is 2-connected, both $G_{1} \cup P_{1} \cup\{f\}$ and $G \backslash\left(G_{1} \cup P_{1} \cup\{f\}\right)$ are flow-admissible, a contradiction. So $G \backslash C$ has exactly three components $C^{\prime}, G_{1}$ and $G_{2}$, that is, (2) holds.

For an $(u, v)$-path $P$ of $G$, we say that $P$ is pendant if $u \in V_{1}(G), v \notin V_{1}(G) \cup V_{2}(G)$ and all internal vertices of $P$ are in $V_{2}(G)$.

Lemma 3.5. Let $H$ be a tree with a unique vertex $C$ of degree at least three, all leaf vertices are unbalanced, and all pendant paths have at most two edges. When $C$ is balanced, $V_{2}(H)=\varnothing$. When $C$ is unbalanced, all degree-2 vertices of $H$ are balanced triangles and leaf vertices that are adjacent to degree-2 vertices are loops. Then $G$ is cover-decomposable or has a 6-cover.

Proof. Assume that the lemma is not true. Since $G$ has a 6 -cover when each component of $G \backslash C$ is a loop, there is a vertex $C^{\prime}$ in $H$ adjacent to $C$ with $\left|C^{\prime}\right| \geqslant 2$. Set $m=$ $\left|V_{G}(C) \cap V_{G}\left(C^{\prime}\right)\right|$. Since $G$ is 2 -connected and $\delta(G) \geqslant 4$, we have $m \geqslant 2$.

We claim that $C^{\prime}$ is balanced or $\left|C^{\prime}\right| \neq 2$. Assume otherwise. Then $C^{\prime}$ is a component of $G \backslash C$ as all degree-2 vertices of $H$ are balanced. Since $\left|C^{\prime}\right|=2$, we have $m=2$. Let $\{u, v\}=V_{G}\left(C^{\prime}\right) \cap V_{G}(C), P_{1}$ and $P_{2}$ be the $(u, v)$-paths of $C$. By Lemma 3.4, $G \backslash C$ has exactly three components $C^{\prime}, G_{1}$ and $G_{2}$, where $G_{1}$ and $G_{2}$ properly intersect $P_{1}$ and $P_{2}$, respectively. When $C \cup G_{1}$ is a necklace, there is a small circuit $D$ of $C \cup G_{1}$ such that $G \backslash D$ is connected. Since $C^{\prime}$ and $G_{2}$ are unbalanced, $G \backslash D$ is flow-admissible, so $G$ is cover-decomposable. Hence, $G_{1}$ is an unbalanced circuit of size at most 2 or $G_{1}$ consists of a balanced triangle and a loop, so is $G_{2}$ by symmetry. By simple computation, $G$ is cover-decomposable or has a 6-cover.

Assume that $C^{\prime}$ is balanced. Then $C^{\prime} \in V_{2}(H)$ is a triangle. So $C$ is unbalanced and $\left|V_{G}\left(C^{\prime}\right) \cap V_{G}(C)\right|=2$ by Lemma 3.1. Let $u, v, P_{1}, P_{2}$ be defined as above. Let $e$ be the loop incident with $C^{\prime}$ and $f$ the edge in $C^{\prime}$ whose ends are $u, v$. Since $C$ is unbalanced, $P_{1} \cup\{f\}$ is balanced and $P_{2} \cup\{f\}$ is unbalanced. Evidently, (a) a component of $G \backslash C$ properly intersects with $P_{1}$, otherwise $P_{1} \cup\{f\}$ and its complement are flow-admissible; and (b) no component of $G \backslash C$ intersects $P_{2}-\{u, v\}$, otherwise the union $G^{\prime}$ of $P_{2} \cup\{f\}$ and all components of $G \backslash C$ intersecting $P_{2}-\{u, v\}$ and $G \backslash G^{\prime}$ are flow-admissible. Then $P_{2} \cup\left(C^{\prime}-\{f\}\right) \cup\{e\}$ and its complement are flow-admissible, a contradiction. 
We may therefore assume that $C^{\prime}$ is unbalanced with $\left|C^{\prime}\right| \geqslant 3$, implying that $C$ is unbalanced by Lemma 3.1. By the choice of $C^{\prime}$, for each component $G^{\prime}$ of $G \backslash C$, either $G^{\prime}$ is a loop or $\left|G^{\prime}\right| \geqslant 3$. When $\left|G^{\prime}\right| \geqslant 3, C \cup G^{\prime}$ is a necklace by Lemma 3.2. Let $D$ be a small circuit of $C \cup C^{\prime}$. Since $G \backslash D$ has two edge-disjoint unbalanced circuits, $G \backslash D$ is disconnected, so a component $G_{D}$ of $G \backslash C$ properly intersects in $C \cap D$. Since $C \cup C^{\prime}$ has three small circuits, $G_{D}$ is the unique component of $G \backslash C$ properly intersecting in $C \cap D$ and $C \cup C^{\prime}$ has exactly three small circuits, implying $\left|C^{\prime}\right|=3$, otherwise $G$ is cover-decomposable. When $G_{D}$ is not a loop, there is a small circuit $D^{\prime}$ of $C \cup G_{D}$ such that $G \backslash D^{\prime}$ is connected, so $G$ is cover-decomposable. Hence, $G_{D}$ is a loop. By the choice of $C^{\prime}$, each component $G^{\prime}$ of $G \backslash C$ that is not a loop is an unbalanced triangle. When $C^{\prime}$ is the unique component of $G \backslash C$ that is not a loop, $G$ has a 3-cover. When there is another component $G_{1}$ of $G \backslash C$ that is not a loop, let $D$ be a small circuit of $C \cup C^{\prime}$ intersecting $G_{1}$. Let $G^{\prime}$ be the union of $D \cup G_{1}$ and the loop incident with $D$. Then $G^{\prime}$ and $G \backslash G^{\prime}$ are flow-admissible, so $G$ is cover-decomposable.

\section{Proof of Theorem 1.2.}

In this section, we prove Theorem 1.2 , which is restated here in a slightly different way.

Theorem 4.1. Every flow-admissible signed Eulerian graph has a 6-cover.

Proof. Assume that the result is not true. Let $G$ be a counterexample with $|V(G)|$ as small as possible. Evidently, the following statements hold.

\subsection{1.}

- $G$ is unbalanced with $\delta(G) \geqslant 4$;

- $G$ has no balanced loops; and

- $G$ is not cover-decomposable, in particular, if $C$ is a non-separating balanced circuit of $G$, then $G \backslash C$ is not flow-admissible.

\subsection{2. $G$ is 2-connected.}

Subproof. Assume otherwise. There are edge-disjoint Eulerian subgraphs $G_{1}, G_{2}$ of $G$ with $\left|E\left(G_{1}\right)\right|,\left|E\left(G_{2}\right)\right| \geqslant 2$, with $\{v\}=V\left(G_{1}\right) \cap V\left(G_{2}\right)$, and with $E(G)=E\left(G_{1}\right) \cup E\left(G_{2}\right)$. Since $G$ is a minimal counterexample and not cover-decomposable, $G_{1}$ and $G_{2}$ are unbalanced. Let $G_{i}^{+}$be a signed graph obtained from $G_{i}$ by adding an unbalanced loop $e_{i}$ incident with $v$ for each integer $1 \leqslant i \leqslant 2$. Since $G_{1}^{+}$and $G_{2}^{+}$are flow-admissible, both of them have 6 -covers by the choice of $G$. Since $\left|V\left(G_{1}\right) \cap V\left(G_{2}\right)\right|=1$, we can obtain a 6 -cover of $G$ by combining 6-covers of $G_{1}^{+}$and $G_{2}^{+}$, a contradiction.

Let $\mathcal{C}$ be an optimal circuit decomposition of $G$ and $H$ the graph determined by $\mathcal{C}$. Since $G$ is connected, so is $H$. By Lemma 2.6, at least two members of $\mathcal{C}$ are unbalanced. Hence, by Lemma 3.2, $|V(H)| \geqslant 3$ and the following holds. If a block of $H$ contains exactly one cut-vertex of $H$, we say the block is a leaf block. 
4.1.3. Each balanced vertex of $H$ is a cut-vertex, in particular, each vertex in a leaf block of $H$ that is not a cut-vertex is unbalanced.

By 4.1.3 or the third part of 4.1.1, for any vertex $C$ of $H$, all components of $G \backslash C$ are unbalanced. For a subgraph $H^{\prime}$ of $H$, each vertex $v \in V\left(H^{\prime}\right)$ is labeled by a circuit $C_{v}$ in $\mathcal{C}$. We say that the subgraph $G^{\prime}=\cup_{v \in V\left(H^{\prime}\right)} E\left(C_{v}\right)$ corresponds to $H^{\prime}$.

4.1.4. Let e be a cut-edge of $H$ whose ends are $C_{i}$ and $C_{j}$. If $e$ is not a leaf edge and $H-\left\{C_{i}, C_{j}\right\}$ has exactly two components, then $C_{i}$ or $C_{j}$ is unbalanced.

Subproof. Assume to the contrary that $C_{i}$ and $C_{j}$ are balanced. Let $G_{1}$ and $G_{2}$ be the subgraphs of $G$ corresponding to the two components of $H-\left\{C_{i}, C_{j}\right\}$ with $V\left(G_{1}\right) \cap$ $V_{G}\left(C_{i}\right) \neq \varnothing$. It follows from 4.1.3 that $G_{1}, G_{2}$ are unbalanced. Moreover, since $G$ is 2-connected, by Lemma 3.1, we have $\left|V_{G}\left(C_{i}\right) \cap V_{G}\left(C_{j}\right)\right|=2$. Let $u \in V_{G}\left(G_{1}\right) \cap V_{G}\left(C_{i}\right)$ and $v \in V\left(G_{2}\right) \cap V_{G}\left(C_{j}\right)$. Since $\left|V_{G}\left(C_{i}\right) \cap V_{G}\left(C_{j}\right)\right|=2$, the graph $C_{i} \cup C_{j}$ has a circuit $C$ avoiding $u$ and $v$ such that $\left(C_{i} \cup C_{j}\right) \backslash C$ is connected up to isolated vertices. Since $H-\left\{C_{i}, C_{j}\right\}$ has exactly two components, $G \backslash C$ is connected, so $G \backslash C$ is flow-admissible. Moreover, since $C_{i} \cup C_{j}$ is balanced by Lemma 3.1, $C$ is balanced, so $G$ is cover-decomposable, a contradiction.

4.1.5. For any separating circuit $C \in \mathcal{C}$, if $G^{\prime}$ is a component of $G \backslash C$ that is not flowadmissible, then one of the following holds.

(1) $G^{\prime}$ is an unbalanced circuit such that $\left|G^{\prime}\right| \leqslant 2$ or $C \cup G^{\prime}$ is a necklace. In particular, when $C$ is balanced, $\left|G^{\prime}\right| \leqslant 2$.

(2) $G^{\prime}$ consists of a loop and a balanced triangle.

Subproof. When $G^{\prime}$ is a circuit, since $\delta(G) \geqslant 4$, by Lemmas 3.1 and 3.2, (1) holds. Assume that $G^{\prime}$ is not a circuit. When $G^{\prime}$ consists of exactly two edge-disjoint circuits that share exactly one vertex, since $C$ only shares vertices with the balanced circuit of $G^{\prime}$ and $\delta(G) \geqslant 4$, the unbalanced circuit $C^{\prime}$ in $G^{\prime}$ has at most two edges. When $\left|C^{\prime}\right|=2$, there is a non-separating balanced circuit of $G$ contained in $C \cup C^{\prime}$, a contradiction. So $C^{\prime}$ is a loop. By Lemma 3.1 and 4.1.2, the balanced circuit in $G^{\prime}$ is a triangle, so (2) holds. Hence, we may assume that $\Delta\left(G^{\prime}\right) \geqslant 6$ or $\left|V_{4}\left(G^{\prime}\right)\right| \geqslant 2$.

Since $G^{\prime}$ is not flow-admissible, by switching we may assume that there is a unique edge $e$ of $G^{\prime}$ labelled by -1 and all other edges in $G^{\prime}$ are labelled by 1 . When $e$ is a loop, let $v$ be the end of $e$, and $B$ a block of $G^{\prime} \backslash\{e\}$ containing $v$, and let $C^{\prime}$ be a circuit of $B$ containing $v$; otherwise, let $\{v\}=\varnothing$, and $B$ the block containing $e$, and let $C^{\prime}$ be a circuit of $B$ with $e \in C^{\prime}$. If possible, we may further assume that $C^{\prime}$ is chosen with $V_{G}\left(C^{\prime}\right) \cap V_{2}\left(G^{\prime}\right) \neq \varnothing$. By Lemma 2.1, there is a circuit $C_{1}$ of $G^{\prime} \backslash \operatorname{loops}\left(G^{\prime}\right)$ with $C^{\prime} \cap C_{1}=\varnothing$ such that $G^{\prime} \backslash C_{1}$ is connected up to isolated vertices. Since $C_{1}$ is balanced and $G \backslash C_{1}$ has two edge-disjoint unbalanced circuits, $G \backslash C_{1}$ is not connected. Hence, $V_{G}(C) \cap V\left(G^{\prime}\right) \subseteq V_{G}\left(C_{1}\right)$ and $\varnothing \neq V_{2}\left(G^{\prime}\right) \subseteq V_{G}\left(C_{1}\right)$ as $e$ is the only edge in $G^{\prime}$ which has a chance to be a loop. By the choice of $C^{\prime}$, the set $V_{2}\left(G^{\prime}\right)$ is contained in another block $B^{\prime}$ of $G^{\prime}$ with $B \neq B^{\prime}$ as $C^{\prime}$ contains no vertex in $V_{2}\left(G^{\prime}\right)$. Since $V_{G}(C) \cap V\left(G^{\prime}\right) \subseteq V\left(B^{\prime}\right)$ and $G$ is 2-connected, $|B|=1$, a contradiction to the choice of $B$. 
4.1.6. For any $C \in \mathcal{C}$, the graph $G \backslash C$ has at most two components.

Subproof. Assume that $G \backslash C$ has three components. Since each component $G^{\prime}$ of $G \backslash C$ is unbalanced and $G \backslash G^{\prime}$ is flow-admissible, $G^{\prime}$ is not flow-admissible. By 4.1.5, $H$ is a tree with $C$ as a unique vertex of degree at least three, and all its pendant paths have at most two edges. When $C$ is balanced, 4.1 .4 implies that $V_{2}(H)=\varnothing$. Hence, by 4.1 .5 and Lemma 3.5, $G$ is cover-decomposable or has a 6 -cover, a contradiction.

4.1.7. For any balanced vertex $C$ of $H$, each degree-1 vertex of $H$ adjacent with $C$ is a loop of $G$.

Subproof. Let $C^{\prime}$ be a degree- 1 vertex of $H$ adjacent with $C$. Assume that $C^{\prime}$ is not a loop of $G$. Then $\left|C^{\prime}\right|=\left|V_{G}(C) \cap V_{G}\left(C^{\prime}\right)\right|=2$ by 4.1.5. It follows from Lemma 3.4 and 4.1.6 that $G$ is cover-decomposable, a contradiction.

4.1.8. $H$ is not a tree.

Subproof. Assume otherwise. By 4.1.6, $H$ is a path. Evidently, at most one vertex in $V_{2}(H)$ is unbalanced, otherwise, $G$ is cover-decomposable. By 4.1.4, no balanced vertices of $H$ are adjacent, so $|V(H)| \leqslant 5$. Moreover, if $|V(H)| \geqslant 4$, then exactly one vertex in $V_{2}(H)$ is unbalanced. Assume that $H$ has two adjacent vertices $C_{1}, C_{2}$ with $\mid V_{G}\left(C_{1}\right) \cap$ $V_{G}\left(C_{2}\right) \mid \geqslant 3$. Then $C_{1} \in V_{1}(H),|V(H)| \leqslant 4$ and $C_{1} \cup C_{2}$ is a necklace by Lemma 3.2. Let $C_{3}$ be the other vertex adjacent to $C_{2}$ in $H$. When $V_{G}\left(C_{2}\right) \cap V_{G}\left(C_{3}\right)$ is in a small circuit of $C_{1} \cup C_{2}$, the graph $G$ has a 6 -cover. When $V_{G}\left(C_{2}\right) \cap V_{G}\left(C_{3}\right)$ is not in a small circuit of $C_{1} \cup C_{2}$, implying $\left|V_{G}\left(C_{2}\right) \cap V_{G}\left(C_{3}\right)\right|=2$, since $V_{G}\left(C_{1}\right) \cap V_{G}\left(C_{3}\right)=\varnothing$, the graph $C_{1} \cup C_{2}$ can be decomposed to two long circuits $C_{1}^{\prime}, C_{2}^{\prime}$, where both share exactly one vertex with $C_{3}$. Note that the circuit decomposition $\left(\mathcal{C}-\left\{C_{1}, C_{2}\right\}\right) \cup\left\{C_{1}^{\prime}, C_{2}^{\prime}\right\}$ is still optimal. Hence, the graph determined by $\left(\mathcal{C}-\left\{C_{1}, C_{2}\right\}\right) \cup\left\{C_{1}^{\prime}, C_{2}^{\prime}\right\}$ is isomorphic to a graph pictured as Figure 1 (c) or (d). Lemma 3.3 implies that $G$ is cover-decomposable or has a 6-cover. Therefore, combined with Lemma 3.1 we can assume that every pair of adjacent vertices in $H$ share at most two vertices in $G$. Note that each degree- 1 vertex of $H$ adjacent to a balanced vertex is a loop by 4.1.7. By simple computation, $G$ has a 6-cover, a contradiction.

4.1.9. $H$ is not 2-connected and whose leaf blocks are isomorphic to $K_{2}$.

Subproof. Assume otherwise. When $H$ is not 2-connected, let $B$ be a leaf block of $H$ that is not isomorphic to $K_{2}$, and $v$ be the unique cut-vertex of $H$ in $V(B)$. When $H$ is 2-connected, let $B=H$ and $v$ any vertex of $B$. By Lemma 2.2, there is an edge $e$ in $B-v$ such that $B-V_{H}(e)$ is connected, so $H-V_{H}(e)$ is also connected. Without loss of generality assume that $C_{1}$ and $C_{2}$ are the ends of $e$. Then $C_{1} \cup C_{2}$ and $G \backslash\left(C_{1} \cup C_{2}\right)$ are connected. Since $C_{1} \cup C_{2}$ is flow-admissible by 4.1.3, the graph $G \backslash\left(C_{1} \cup C_{2}\right)$ is not flow-admissible. Since $H$ is not isomorphic to the graph pictured as Figure 1 (d) by Lemma 3.3, $H$ has exactly three unbalanced vertices and exactly two leaf blocks, one of which is $B$ that is isomorphic to $K_{3}$ and the other is isomorphic to $K_{2}$. Let $C_{1} C_{2} C_{3} \ldots C_{n}$ be a longest path in $H$. It follows from 4.1.4 that $n=4$. By 4.1.7, the circuit $C_{4}$ is a 
loop of $G$. That is, $H$ is isomorphic to the graph pictured as Figure 1 (c). Hence, $G$ is cover-decomposable or has a 6-cover by Lemma 3.3, a contradiction.

Let $B$ be a block of $H$ with $|V(B)| \geqslant 3$. By 4.1.8 and 4.1.9, such $B$ exists and $B$ is not a leaf block. When $H$ has two blocks that are not isomorphic to $K_{2}$, it follows from 4.1.3 and 4.1.9 that $G$ is cover-decomposable. Hence, $B$ is the unique block of $H$ that is not isomorphic to $K_{2}$. By 4.1.3, each vertex in $B$ that is not a cut-vertex of $H$ is unbalanced.

Let $u \in V(B)$ be a cut vertex of $H$. When $u$ is unbalanced or $H$ has two pendant paths using $u$, let $H_{1}$ be the union of all pendant paths containing $u$, and $G_{1}$ the subgraph of $G$ corresponding to $H_{1}$. Since $|V(B)| \geqslant 3$, by 4.1.3 and 4.1.9, both $G_{1}$ and $G \backslash G_{1}$ are flow-admissible, a contradiction. Hence, $u$ is balanced and $H$ has exactly one pendant path using $u$. By the arbitrary choice of $u$, all cut-vertices of $H$ in $B$ are balanced. Using a similar strategy, all vertices in $V_{2}(H)-V(B)$ are balanced. Combined with 4.1.4, we have $V_{2}(H)-V(B)=\varnothing$. That is, each pendant path of $H$ has exactly one edge. By 4.1.7, each vertex in $V_{1}(H)$ is a loop of $G$.

When there is a vertex in $V(B)$ that is not a cut-vertex of $H$, let $v$ denote such a vertex. Otherwise, let $v$ be any vertex of $B$. By Lemma 2.2, there is an edge $e \in B-v$ such that $B-V(e)$ is connected. Let $H_{1}$ be the union of $e$ and all pendant paths of $H$ using an end of $e$, and $G_{1}$ be the subgraph of $G$ corresponding to $H_{1}$. Since each vertex in $B$ that is not a cut-vertex of $H$ is unbalanced, $H_{1}$ contains two unbalanced vertices, so $G_{1}$ is flow-admissible. Since $H-V\left(H_{1}\right)$ is connected and has an unbalanced vertex, $H$ is isomorphic to a graph pictured as Figure 1 (a) or (b). Lemma 3.3 implies that $G$ is cover-decomposable or has a 6-cover, a contradiction.

\section{Acknowledgements}

We thank the referees for their careful reading of this manuscript and their detailed comments.

\section{References}

[1] J. C. Bermond, B. Jackson, F. Jaeger, Shortest covering of graphs with cycles, J. Combin. Theory Ser. B, 35:297-308, 1983.

[2] R. Chen, M. DeVos, D. Funk, I. Pivotto, Graphic representation of graphic frame matroids, Graphs and Combin., 31:2075-2086, 2015.

[3] J. Cheng, Y. Lu, R. Luo, C. Q. Zhang, Shortest circuit cover of signed graphs, J. Combin. Theory Ser. B, 134:164-178, 2019.

[4] G. Fan, Integer flows and cycle covers, J. Combin. Theory Ser. B, 54:113-122, 1992.

[5] G. Fan, Flows and circuit covers in signed Graphs, Lectures in NSFC Tianyuan Summer School, Jinhua, 2018.

[6] E. Máčajová, M. Škoviera, Nowhere-zero flows on signed Eulerian graphs, SIAM J. Discrete Math., 31:1937-1952, 2017. 
[7] T. Zaslavsky, Biased graphs. I. Bias, balanced, and gains, J. Combin. Theory Ser. B, 47:32-52, 1989.

[8] T. Zaslavsky, Biased graphs. II. The three matroids. J. Combin. Theory Ser. B, 51:46-72, 1991. 